\title{
Aerodynamic Characteristics of Dragonfly Wing Corrugation in Hovering Flight
}

\author{
Chao WANG, Peng XIE, Chao-Ying ZHOU* and Rui ZHANG \\ Shenzhen Graduate School, Harbin Institute of Technology, 518055, Shenzhen, China \\ *cyzhou@hit.edu.cn
}

Keywords: Corrugated wing; Hovering wing; Aerodynamic performance; Numerical simulation.

\begin{abstract}
In this study, the aerodynamic characteristics of dragonfly wing corrugation in hovering flight are investigated using the computational fluid dynamics (CFD) methods. Three corrugated cross sections of the right forewing of a dragonfly (Aeshna cyanea) are considered. The effects of wing corrugation are identified by comparing the aerodynamic differences between the corrugated and the corresponding smoothed wing sections, and the underlying flow mechanisms of the corrugation effects are revealed by analyzing the flow fields and surface pressure distributions of the wings. The results show that the wing corrugation has little effects on aerodynamic performance of wings. The time histories of aerodynamic forces of the corrugated wings are very close to those of the smoothed wings. The flow information indicates that the corrugated and smoothed wings have approximately the same vorticity around the wings.
\end{abstract}

\section{Introduction}

Recently, the aerodynamic performance of flying insects is gaining more attention because of the possible applications in Micro Aerial Vehicles (MAVs). Much work has been done on aerodynamic characteristics of insect flapping wings using experimental and computational methods and considerable understandings of the aerodynamic mechanisms have been achieved [1-4].

It is well known that most of insect wings are corrugated. In aforementioned studies, ellipse sections, flat plates and traditional airfoils were employed as model wings. The authors have implicitly assumed that the corrugation has minor effects on aerodynamic performance of the wings. It is of great interest to test this assumption, and to know whether the wing corrugation significantly influences the aerodynamic performance of flying insects.

To investigate the unsteady aerodynamic forces of corrugated wings in flapping flights, Premanchandran [5] analyzed the aerodynamic characteristic of corrugated wings at low Reynolds number in hovering flight. Their results showed that the lift force generated by the corrugated airfoil was a little larger than that of the NACA0012 airfoil. Meng et al. [6,7] compared the aerodynamic characteristic of corrugated airfoils with the flat plate under the forward flight and hovering flight conditions. Their results showed that the corrugation decreased the aerodynamic forces slightly, and using a flat plate wing to model the corrugated wing was a good approximation.

Considering that the wing models in Meng et al. [6,7] have extremely different contour thickness, which may have significant effects on aerodynamic performance of flapping wings, the effects of wing corrugation on its flight performance is still not fully understood. In this paper, a numerical study on aerodynamic characteristics of dragonfly wing corrugation in hovering flight is carried out. Three corrugated cross sections of the right forewing of a dragonfly (Aeshna cyanea) are considered. The effects of wing corrugation are identified by comparing the aerodynamic differences between the corrugated and smoothed wing sections. The corrugation effects on aerodynamic forces, the energy consumption and flow field of the wings are examined in detail

\section{Problem Descriptions}

To clarify the effects of varied corrugated sections along the longitudinal axis of dragonfly wings, three corrugated cross sections at $0.3 L_{r e f}, 0.5 L_{r e f}$ and $0.7 L_{r e f}$ of the right forewing of a dragonfly 
(Aeshna cyanea) are digitally extracted from the experiments of Kesel [8] and shown in Figure 1, where $L_{\text {ref }}$ is the relative span length. Three pairs of wing profiles are compared in present studies, where the pleats of each corrugated airfoil are filled with straight lines, forming the enveloped airfoil. The thickness of corrugated airfoils are fixed at $1 \% c$, where $c$ is the chord length. The chord lengths of each airfoil are equal.

The wing undergoes the combined translational and rotational motions. The schematic of wing motion is drawn in Figure 2, where a thick line with a circle at one end is used to represent the wing section and the circle indicates the leading edge of the wing. The dashed line shows the stroke plane which has an inclined angle of $\beta$.
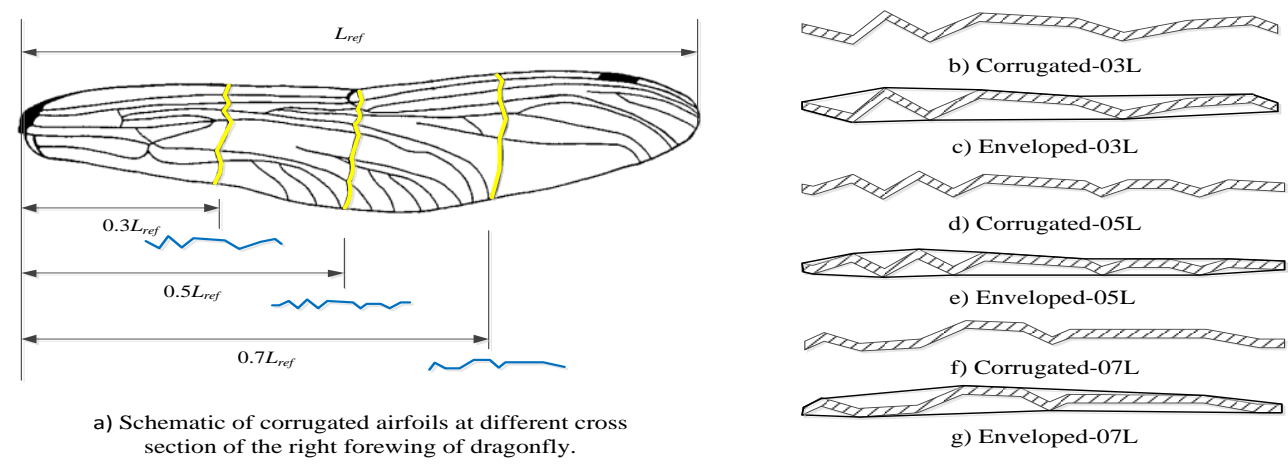

Fig. 1. Schematic of corrugated and enveloped airfoils.

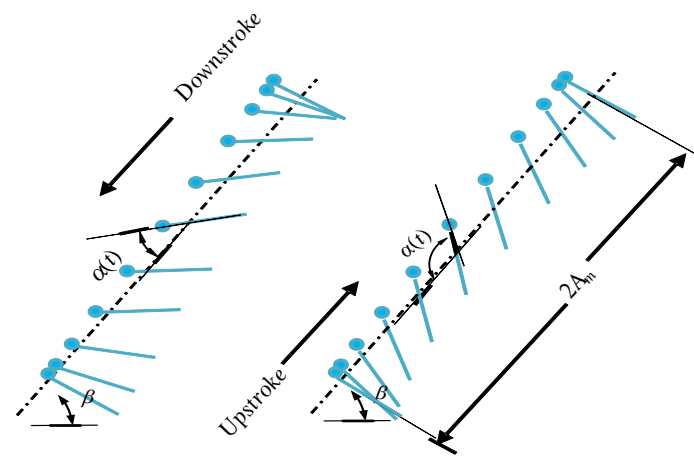

Fig. 2. Schematic of the flapping motion.

The flapping wing motions can be described as:

$$
\begin{gathered}
A(t)=A_{m} \cos (2 \pi f t), \\
\alpha(t)=\alpha_{0}-\alpha_{m} \sin (2 \pi f t) .
\end{gathered}
$$

Where $A(t)$ and $\alpha(t)$ are the translational displacement and rotational angle, respectively. $A_{m}$ and $\alpha_{m}$ are amplitudes of the translational and rotational motion, respectively. $\alpha_{0}$ is the initial rotational angle and $f$ is the flapping frequency.

\section{Numerical Methods}

\section{Governing Equation of Fluid Flow}

Considering the flight characteristics of insects in nature, the flow around the wing is assumed to be laminar and incompressible. The two-dimensional Navier-Stokes equations are applied in the present study. The equations can be written as the following form:

$$
\nabla \cdot \boldsymbol{u}=0
$$




$$
\frac{\partial \boldsymbol{u}}{\partial \tau}+\boldsymbol{u} \cdot \nabla \boldsymbol{u}=-\nabla p+\frac{1}{R e} \nabla^{2} \boldsymbol{u}
$$

where $\mathbf{u}, p$ and $\tau$ are the non-dimensionalized flow velocity, fluid pressure and time, respectively. $\operatorname{Re}$ represents Reynolds number and $\operatorname{Re}=\rho U_{\text {ref }} / \mu$, where $\rho$ is the fluid density, $\mu$ is the viscosity, and $U_{r e f}$ is the maximum translating velocity. Finite volume method is introduced for spatial discretization. Second-order accurate upwind scheme and second-order central difference scheme are applied in the discretizations of convective and diffusive terms respectively. The decoupling between the pressure and the velocity is achieved through the use of SIMPLEC algorithm. The lift and thrust coefficients are calculated by $C_{L}=F_{L} /\left(0.5 \rho U_{r e f}^{2} c\right)$ and $C_{T}=-F_{D} /\left(0.5 \rho U_{r e f}^{2} c\right)$, respectively, where $F_{L}$ and $F_{D}$ are the lift and drag forces. The energy consumption is determined by :

$$
P(t)=-\left[F_{S} \cdot V(t)+M \cdot \dot{\alpha}(t)\right] .
$$

Where $M$ is the aerodynamic moment and $F_{S}$ is the resultant force along the stroke direction. Then the energy coefficient is defined by $C_{P}=P(t) /\left(0.5 \rho U_{r e f}{ }^{3} c\right)$. The time-averaged values of the lift, thrust and energy coefficients are expressed by $C_{L m}, C_{T m}$ and $C_{P m}$ respectively. The lifting efficiency can be calculated by $\eta_{L}=C_{L m} / C_{P m}$.

A schematic diagram of computational domain is shown in Figure 3. Structured quadrilateral cells are used in the zone $\Omega_{1}$ to encompass the whole wing movement with the predefined motion, while unstructured triangular cells are used in the zone $\Omega_{2}$ to meet the requirement of grid remeshing at each time step. Non-slip wall boundary condition is applied at the surface of the wing $\Gamma_{1}$ and a uniform boundary condition is adopted at the outer boundary of $\Omega_{2}$ considering that $\Gamma_{2}$ is far enough from the wing so that the fluid flow can be considered to be steady and uniform. The uniform boundary condition is given as:

$$
p=p_{\infty}, \frac{\partial \boldsymbol{u}}{\partial n}=0
$$

where the $p_{\infty}$ is the standard atmospheric pressure and $n$ is the outward normal unit vector at the outer boundary.

\section{Validation of Numerical Method}

To examine the reliability of the numerical methods, a typical hovering motion studied by Wang et al. [9] is tested. The flapping motion follows the Eqns.(1) and (2). The wing section is simplified as a ellipse with thickness ratio 0.125 . The kinematic parameters of the motion are given as: $R e=157$, $A_{m}=1.25 c, \alpha_{0}=7 \pi / 12, \alpha_{m}=\pi / 4, \beta=\pi / 3$. The rotation center is at the point of $0.5 c$ away from the leading edge. The time histories of the lift and drag coefficients of the wing are shown in Figure 4 where some results from Refs. [10,11] with the same wing kinematics are also presented for comparison. It is seen that the variation of the lift and drag coefficients in present studies are in good agreement with the previous studies.

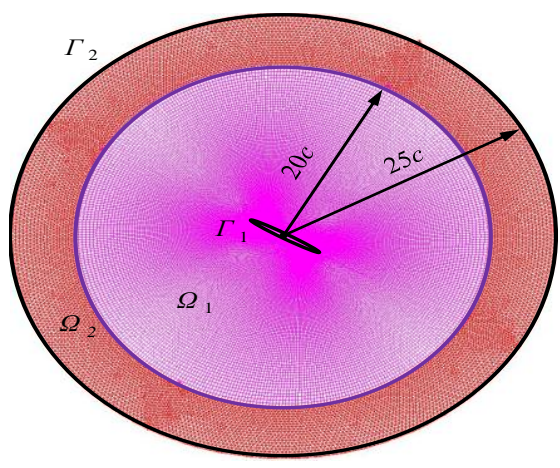

Fig. 3. Schematic of the computational domain. 


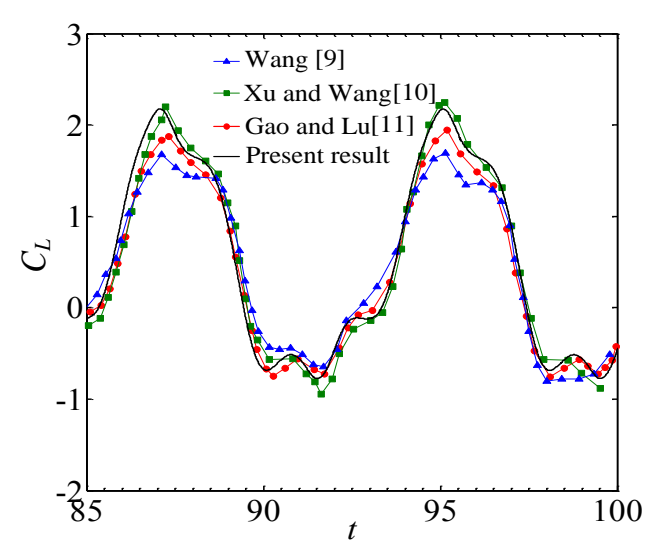

a) Lift coefficient

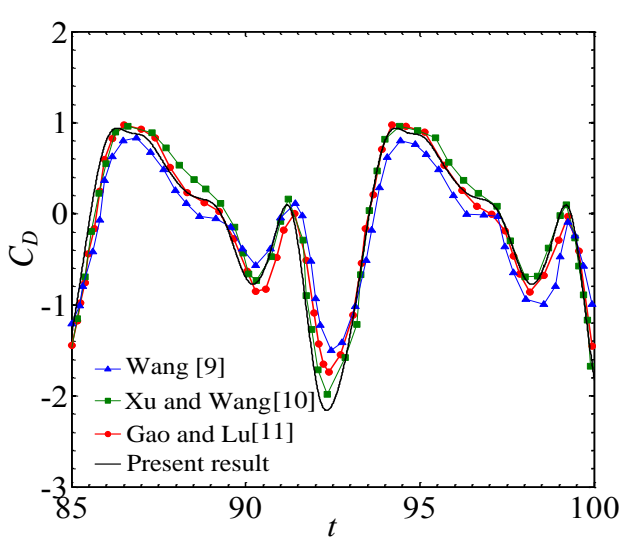

b) Drag coefficient

Fig. 4. Comparison of force coefficients between the present results and the previous studies.

\section{Results and Discussion}

To study the aerodynamic characteristics of dragonfly wing corrugation during the hovering flight, the following parameters are fixed where $R e=1000, A_{m}=1.25 c, \alpha_{0}=\pi / 2, \alpha_{m}=\pi / 4, \beta=\pi / 3$. The rotation center is at the point of $0.25 c$ away from the leading edge. In Table 1 , the time-averaged aerodynamic performance of the corrugated airfoils and the corresponding enveloped airfoils are presented. It is seen that the lift forces and lifting efficiency in corrugated wings are litter larger than those in the corresponding enveloped wings. The maximum performance improvements due to the corrugation not exceed $2.5 \%$.

Table 1. Comparison of the aerodynamic performance between the corrugated airfoils and the enveloped airfoils.

\begin{tabular}{|c|c|c|c|}
\hline Wing model & $C_{L m}$ & $C_{P m}$ & $\eta_{L}$ \\
\hline Corrugated-03L & 0.9446 & 3.1068 & 0.3040 \\
\hline Enveloped-03L & 0.9237 & 3.0627 & 0.3016 \\
\hline Corrugated-05L & 0.9424 & 3.1784 & 0.2965 \\
\hline Enveloped-05L & 0.9261 & 3.1054 & 0.2982 \\
\hline Corrugated-07L & 0.9335 & 3.0503 & 0.3060 \\
\hline Enveloped-7L & 0.9272 & 3.0508 & 0.3039 \\
\hline
\end{tabular}

In order to analyze the details of the effects of corrugation on aerodynamic forces of wings during the hovering flight, the time histories of $C_{L}$ and $C_{P}$ for the airfoils Corrugated-05L and the Enveloped-05L during one flapping cycle are shown in Figure 5. It is shown that the difference between two airfoils in the aerodynamic forces and energy consumption are very small.

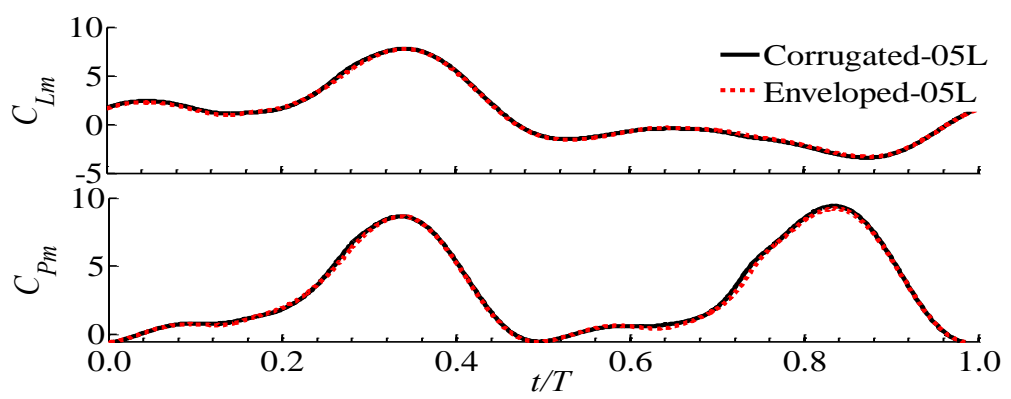

Fig. 5. The time histories of lift $\left(C_{L m}\right)$ and energy $\left(C_{P m}\right)$ coefficients of the corrugated airfoil 05L and enveloped airfoil 05L during a complete flapping cycle.

Figure 6 gives the pressure distributions of the airfoils Corrugated-05L and the Enveloped-05L at various times during a flapping cycle. It can be seen that the curves of $C_{p}{ }^{*}\left(C_{p}{ }^{*}=\left(p-p_{\infty}\right) /\left(0.5 \rho U_{r e f}{ }^{2} c\right)\right)$ 
for two airfoils nearly have the same trend, except that in the region of the pleats, the surface pressure of the corrugated wing varies around that of the enveloped wing. That is, the corrugation only produces some local "waves" in the surface pressure distribution, hence it has litter effect on the aerodynamic forces.

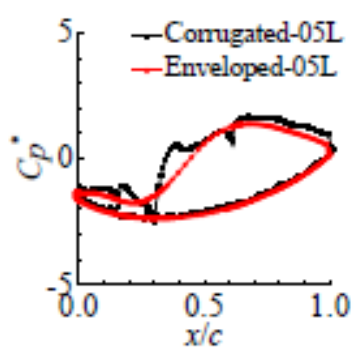

a) $0 T$

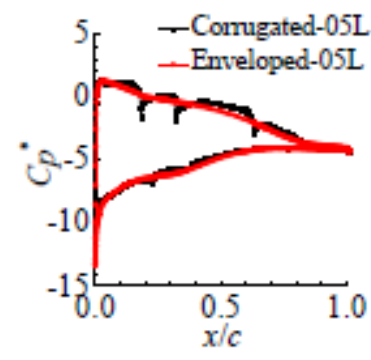

b) $0.25 T$

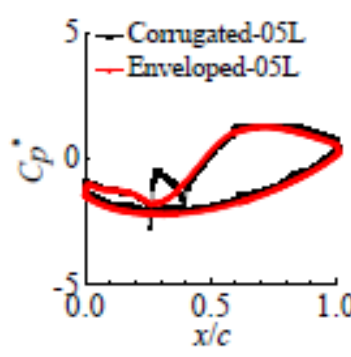

c) $0.50 T$

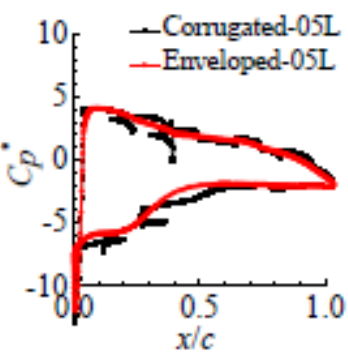

d) $0.75 T$

Fig. 6. The pressure coefficient distribution of airfoils Corrugated-05L and Enveloped-05L during a complete flapping cycle.

Figure 7 shows the vorticity contours of the airfoils Corrugated-05L and the Enveloped-05L at different times during one flapping cycle. It is shown that both in downstroke and upstroke, the flow is separated with a leading edge vortex (LEV) attaching to the upper wing surface. The positive (or negative) vorticity shed from the trailing edge of the corrugated wing is almost the same as that of the enveloped wing. Since the total vorticity is conserved, this indicates that the corrugated wing and the enveloped win have approximately the same total negative (or positive) vorticity around the wing. On the basis of the relation between the aerodynamic force and the vorticity moment, the two wings would have approximately the same aerodynamic force, as is shown in Table 1 and Figure 5.

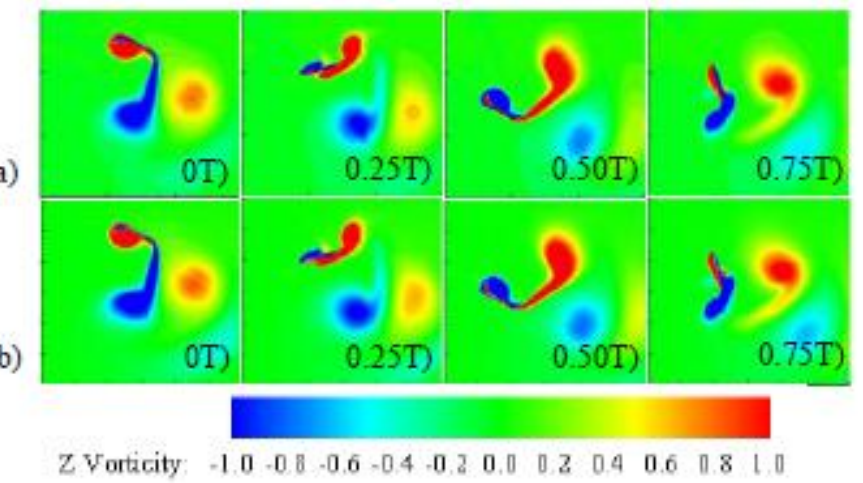

Fig. 7. Contour of vortices of airfoils during a complete flapping cycle.

a) Corrugated-05L ; b) Enveloped-05L.

\section{Conclusion}

The aerodynamic characteristics of corrugated dragonfly wings under hovering flight are investigated using the CFD methods. Three corrugated cross sections of the right forewing of a dragonfly (Aeshna cyanea) are considered. The effects of wing corrugation are identified by comparing the aerodynamic differences between the corrugated and the corresponding smoothed wings. The results show that the time histories of aerodynamic forces of the corrugated wings are very close to those of the smoothed wings. The corrugation increases lift force slightly. The changes in lift force and lifting efficiency resulting from the corrugation are no more than $2.5 \%$. The flow information indicates that the corrugated and smoothed wings have approximately the same vorticity around the wings. 


\section{Acknowledgement}

The work described in this paper was supported by Shenzhen Science and Technology Plan Project (Grant No. ZDSYS2014050816154782 9).

\section{References}

1. C. P. Ellington, C. VandenBerg, A. P. Willmott, ALR Thomas, Leading-edge vortices in insect flight, Nature, 384 (1996), pp.626-630

2. M. H. Dickinson, F. O. Lehmann, S. P. Sane, Wing rotation and the aerodynamic basis of insect flight, Science, 284 (1999), pp.1954-1960

3. M. Sun, J. Tang, Unsteady aerodynamic force generation by a model fruit fly wing in flapping motion, J Exp Biol, 205 (2002), pp.55-70

4. Z. J. Wang, J. M. Birch, M. H. Dickinson, Unsteady forces and flows in low Reynolds number hovering flight: two-dimensional computations vs robotic wing experiments, J Exp Biol, 207 (2004), pp.449-460

5. S. Premachandran, M. Giacobello, The effect of wing corrugations on the aerodynamic performance of low-Reynolds number flapping flight, 17th Australasian Fluid Mechanics Conference, (2010), Auckland, New Zealand

6. X. Meng, M. Sun, Aerodynamic effects of corrugation in flapping insect wings in forward flight, J Bionic Eng, 8 (2011), pp.140-150

7. X. G. Meng, L. Xu, M. Sun, Aerodynamic effects of corrugation in flapping insect wings in hovering flight, J Exp Biol, 214 (2011), pp.432-444

8. A. B. Kesel, Aerodynamic characteristics of dragonfly wing sections compared with technical aerofoils, J Exp Biol, 203 (2000), pp.3125-3135

9. Z. Jane Wang, Two dimensional mechanism for insect hovering., Phys Rev Lett, 85 (2000), pp.2216-9

10. S. Xu, Z. Jane Wang, An immersed interface method for simulating the interaction of a fluid with moving boundaries, J Comput Phys, 216 (2006), pp.454-493

11. T. Gao, X. Y. Lu, Insect normal hovering flight in ground effect, Phys Fluids, 20 (2008), pp.087101 